\title{
Tiered Approach to Resilience Assessment
}

\section{AUTHORS:}

Igor Linkov $^{1 *}$, Cate Fox-Lent ${ }^{1}$, Craig R. Allen ${ }^{2}$, James C. Arnott ${ }^{3,11}$, Emanuele Bellini ${ }^{4}$, Jon Coaffee $^{5}$, Marie-Valentine Florin ${ }^{6}$, Kirk Hatfield ${ }^{7}$, Iain Hyde ${ }^{22}$, William Hynes ${ }^{8}$, Aleksandar Jovanovic $^{9}$, Roger Käsperson ${ }^{10}$, John Katzenberger ${ }^{11}$, Patrick W. Keys ${ }^{12,23}$ James H. Lambert $^{13}$, Richard Moss ${ }^{14}$, Peter S. Murdoch ${ }^{15}$, Jose Palma-Oliveira ${ }^{16}$, Roger S. Pulwarty ${ }^{17}$, Laura Read ${ }^{1}$, Dale Sands ${ }^{17}$, Edward A. Thomas ${ }^{19}$, Mari R. Tye ${ }^{20}$, David Woods ${ }^{21}$

${ }^{1}$ United States Army Corp of Engineers, USA

${ }^{2}$ US. Geologicat Survey, Nebraska Cooperative Fish \& Wildlife Research Unit, University of Nebraska-Lincoln, USA

${ }^{3}$ University of Michigan, USA

${ }^{4}$ University of Florence, Italy

${ }^{5}$ University of Warwick, UK

${ }^{6}$ International Risk Governance Council, Switzerland

${ }^{7}$ University of Florida, USA

${ }^{8}$ Future Analytics Consulting, Ireland

${ }^{9}$ Steinbeis Advanced Risk Technologies, Germany

${ }^{10}$ Clark University, USA

${ }^{11}$ Aspen Global Change Institute, USA

${ }^{12}$ Stockholm Resilience Centre, Sweden

${ }^{13}$ University of Virginia, USA

${ }^{14}$ Joint Global Change Research Institute, USA

${ }^{15}$ United States Geological Survey, USA

${ }^{16}$ University of Lisbon, Portugal

${ }^{17}$ National Oceanographic and Atmospheric Administration, USA

${ }^{18}$ AECOM Environment, USA

${ }^{19}$ Natural Hazard Mitigation Association, USA

${ }^{20}$ National Center for Atmospheric Research, USA

${ }^{21}$ Ohio State University, USA

${ }^{22}$ State of Colorado, USA

${ }^{23}$ Colorado State University, USA

*e-mail: 1gor.linkov@usace.army.mil

This is the author manuscript accepted for publication and has undergone full peer review but has not been through the copyediting, typesetting, pagination and proofreading process, which may lead to differences between this version and the Version of Record. Please cite this article as doi: 10.1111/risa.12991.

This article is protected by copyright. All rights reserved. 


\section{ABSTRACT}

Regulatory agencies have long adopted a three-tier framework for risk assessment. We build on this structure to propose a tiered approach for resilience assessment that can be integrated into the existing regulatory processes. Comprehensive approaches to assessing resilience at appropriate and operational scales, reconciling analytical complexity as needed with stakeholderneeds and resources available, and ultimately creating actionable recommendations to enhance resilience are still lacking. Our proposed framework consists of tiers by which analysts can select resilience assessment and decision support tools to inform associated management actions relative to the scope and urgency of the risk and the capacity of resource managers to improve system resilience. The resilience management framework proposed is not intended to supplant either risk management or the many existing efforts of resilience quantification method development, but instead provide a guide to selecting tools that are appropriate for the given analytic need. The goal of this tiered approach is to intentionally parallel the tiered approach used in regulatory contexts so that resilience assessment might be more easily and quickly integrated into existing structures and with existing policies.

\section{KEYWORDS}

resilience, disaster preparedness, systems analysis, business processes, policy analysis, risk analysis

\section{INTRODUCTION}

The concept of resilience has become prevalent among scientists, engineers, and planners in a range of disciplines in the socio-ecological fields (e.g. ecology, urban planning, flood protection, drought management) and across public domains (e.g. city managers, state, regional, and federal officials). The private sector, government, and society have considered 
its application to problems such as disruption from climate change and the challenge of ecosystem management (United Nations, 2015; Walker et al. 2004). However; the term itself carries such a broad range of meanings (Gallopin, 2006; Angeler and Allen, 2016; Quinlan et al., 2015, that it can be difficult to validate or generalize what effective resilience means in practice (Moser and Boykoff 2013). A diversity of definitions of, and underlying rationales for, resilience can confound planning, implementation, and monitoring (Linkov and Florin, 2016; Larkin et al., 2015). In fact, it may be that different fields eventually adopt different conceptualizations of resilience, whether that be the ability to rebound to a previous state, to fail gracefully, or sustain adaptation (Woods, 2006, Linkov and Palma-Oliveira, 2017). Nonetheless, the appeal of resilience has persisted due to the perceived failure of risk management with respect to some of the world's emerging challenges. Myriad tools and methods marketed as resilience assessments now exist, but take very different formats (Florin and Linkov, 2016; Nordgren, Stults and Meerow, 2016; Arnott, Moser, and Goodrich, 2016). Some are as simple as a checklist, others are geo-spatial visualizations of quantifiable metrics, while still others are network modeling methods but with no generalized form, they are custom built for each application. The outputs of these tools are similarly varied, including maps, scores, and performance-time graphs. Developers of the tools span a wide range of entities including academic, private (e.g., consulting), program sponsors (e.g., foundations and agencies), boundary organizations that bridge across research, policy, and practitioner realms, and users themselves. Potential users include state and city managers, industry process administrators, and utility operators, many lacking the expertise to choose among the rapidly accumulating products in this emerging field. There are already several calls for US federal agencies to implement resilience and the need for guidance on how the various methods and approaches to resilience assessment can be synthesized into a regulatory policy will only grow. 
We recognize that there is ongoing discussion of the meaning of resilience and of the specific differences between risk and resilience. For our part we make the following assumptions: Although several approaches to risk assessment exist, the methods adopted by US regulatory agencies are largely based around the risk $=$ threat $x$ vulnerability $x$ consequence model (National Research Council 1983). Whenever risks are identified in this quantitative manner and actions taken to reduce risk, there still remains residual risk. As such, resilience assessment and management is, in part, an effort to address that remaining known, but unmitigated, risk as well as enhance the overall ability of the system to respond to unknown or emerging threats. One of the biggest challenges to effective risk assessment and management is cost. If a Tier I risk assessment indicates any risks of concern, a Tier II or Tier III risk assessment generally involves significant cost and time to collect the relevant data. At the same time, once unacceptable risks are clearly identified, the cost to replace products, harden the system, or change operational procedure is also resource intensive. By integrating resilience assessment with risk assessment, the risk management requirements may be reduced or the same process may also be used to identify resilience enhancements that help manage residualrisk. We propose a tiered approach to resilience assessment that can be integrated with similar tiered approaches adopted by many US regulatory agencies for risk assessment in order to ease the way for policy development, open a pathway to more widespread adoption of resilience practices, and enhance the current risk assessment processes.

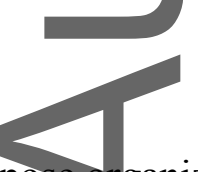

We propose organizing resilience assessment tools into three tiers to provide a structure that allows regulatory agencies to incorporate resilience assessment with existing risk assessment protocols. We choose this framework because it is similar to tiered approaches that initially 
allowed risk analysis to be used for regulatory purposes (WHO 2008; Özkaynak, Frey, and Hubbell 2008). In that implementation, low-level tiers are for screening and use traditional default-based deterministic methods for analysis. These are normally conservative, lower cost, and most useful for identifying cost-effective and achievable actions to reduce risk (USEPA, 2014). Progression from low tiers to higher tiers occurs when risk is near or above accepted thresholds. The specific conditions that result in high risk scenarios must be investigated to develop targeted risk management. Higher tier analytical approaches are also relevant whendecision stakes are high, success is a matter of probability, and debate between options is contested. Many regulatory and standards organizations use similar strategies for risk assessment in order to balance risk understanding with cost. For example, the American Society for Testing and Materials (ASTM) provides a three-tier process for decision making on selecting risk-based corrective action at chemical release sites. If corrective action goals are met in the Tier I analysis, no further work is needed, if not, data needs for Tier II must be identified, and so on (ASTM 2015). The European Food Safety Authority utilizes a tiered approach in toxicological studies. Tier I assesses absorption toxicokinetics and sensitive groups (in vitro genotoxicity). Only if the food additive shows specific levels of availability and toxicity is further study undertaken (Gott 2012). The US Federal Emergency Management Agency (FEMA) Handbook for the Seismic Evaluation of Buildings consists of a screening phase, evaluation phase, and detailed evaluation phase. At any tier, the assessor can report deficiencies and recommend mitigation or proceed to the next tier to conduct further evaluation to resolve uncertainty (FEMA 1998). The US Nuclear Regulatory Commission (NRC) has identified a three-tier approach to evaluate risk associated with proposed changes to plant-specific technical specifications. Tier I assesses change in core damage frequency, Tier II identifies potentially high risk configurations, and, if necessary, Tier III guides development of a risk management program (USNRC 2009). 
Similarly, resilience can be investigated in a tiered manner. We present a framework to organize the various quantitative resilience assessment methods that are available for local, regional, national and global agencies seeking to reduce risk and enhance recovery capacity from risk events. We describe how this framework can contribute to practical ways for resources managers to assess changes in resilience now. An outline of this approach has been presented in the International Risk Governance Council Resource Guide on Resilience (Linkov and Fox-Lent 2016). Here we more fully discuss the role of tiers in resilience assessment and offer a general approach to operationalize quantitative resilience assessment for any discipline.

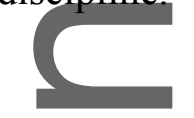

\section{KEY FEATURES OF A TIERED FRAMEWORK FOR RESILIENCE ASSESSMENT}

An overview of the tiered approach is shown in Figure 1. The goal of each tier is to describe the performance and relationship of critical systems in order to identify management options that enhance performance in parallel with activities that reduce risk. The methods of Tier I quickly and inexpensively identify the broad functions that a system provides to human society or the environment and prioritize the performance of the critical functions both during and in the time following a disruptive event. Analytically, this framing and characterization analysis makes use of existing data, expert judgment, and conceptual models. The methods of Tier II describe the general organization and relationships of the system in a simple form such as a process model or critical path model. Identifying sequential and parallel events in a disturbance can reveal feedback processes and dependencies that are the root of cascading system failures. The methods of Tier III build a detailed model of important functions and related sub-systems where each process and each component of the system is parameterized. 
The process can be halted at any tier when enough information has been synthesized such that actionable system investments or projects to improve system resilience, given available resources, have been identified by the decision makers.

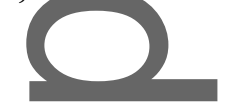

The resilience management framework proposed below is not intended to supplant either risk management or the many existing efforts of resilience quantification method development, but instead provide a guide to selecting tools that are appropriate for the given analytic need. The goal of this tiered approach is to intentionally parallel the tiered approach used in regulatory contexts so that resilience assessment might be more easily and quickly integrated into existing structures and with existing policies, rather than require the time-consuming effort of changing legislation and selling a "new" concept altogether. We highlight these similarities and differences for this purpose of making resilience assessment seem readily accessible for regulatory agencies.

\subsection{Tier I: Screening Level}

\subsubsection{Approach}

A Tier I assessment establishes the role of particular components or actions of interest within the larger context of community, industry, or environment. Regardless of the final question to be targeted (e.g. select from alternatives A, B, or A+B), it is critical to understand the larger system within which a process or entity operates, including the ecology, climate, infrastructure, policies, and human behaviors that govern the performance of the system. Resilience is a property of functions and systems, not features and so at this level events will be considered through their performance within the overall system, not just their performance with respect to a specific feature. For example, while one could do a risk assessment of a reservoir, resilience assessment should look at what function the reservoir plays within a 
larger flood management, water provisioning and/or environmental system and understand what the performance or reduction in performance of the reservoir might mean to the system as a whole. Tier I seeks to identify the major social-ecological-technological properties of the system and to prioritize the critical functions. One aspect of Tier I assessment is to consider not just direct threats to the target system itself, but how disruptions in associated systems may changethe demand on the system of interest.

\subsubsection{Methods}

Existing tools useful for Tier I include screening level assessments based on resilience indices or scorecards developed from formalized libraries of existing metrics or surveys (e.g. UNISDR, 2014; NOAA, 2007; Nemec et al. 2014; Cutter et al. 2010; Flanagan et al. 2011; Peacock et a1. 2010; Sands 2015). In general, Tier I approaches should help the user organize existing information and data to provide a big-picture view of the system. The appropriate tools have the following characteristics: simple system representation, easy consensus on major criteria from stakeholders, retrospective in considering historical records, and conservative in assumptions about the future. However, Tier I approaches rely on indicators of a system, rather than models of a system and so methods should be carefully selected based on the stated goal or intent of the developers (Bakkensen et al. 2016).

2.1.3. Outcomes

In a Tier Irisk assessment, a survey of potential risks is compared to accepted thresholds to determine whether further analysis is required. Here the results of Tier I resilience tools are often relative rather than absolute, whether relative to a similar location that used the same assessment, or relative to the same location at a previous point in time. Thresholds for "good" and "bad" resilience performance do not yet exist, but if the output indicates that the system already performs better than some statistically determined portion of the country, or shows improvement over a previous assessment by some acceptable amount, further analysis may 
be determined to be unnecessary or a lower priority. The main goal of Tier I, however, is to identify and prioritize the critical functions of the system. Whether further analysis is needed (1)

or not, Tier I assessments help decision makers identify "easy wins," or investments in some part of the system that can significantly improve overall resilience and that come at minimal cost or debate. If it is determined that further action is needed in order to identify controlling processes and the efficacy of management actions, Tier I assessments help pare down the sectors to just those critical system functions to be maintained during and following a disruptive event and are taken forward into the Tier II analysis. Once this approach is used to identify the critical functions and their relationship to other features in the system, the effort in Tiers II or III could be used to examine management alternatives in more detail.

\subsection{Tier II: Process Flow Model}

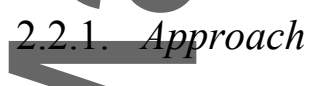

Tier II introduces descriptions of the structure of the system. These might be simple process diagrams or flowcharts that indicate some relationship between system components in time or space and describe major feedbacks within the system or connections to other systems. The Tier I outputs can be used to determine what can be left out of further modeling efforts but still allow evaluation of the salient parts of alternative projects in Tier II. (A similar determination can be done when moving from Tier II to Tier III.) Any model is necessarily imperfect and incomplete; the Tier II model should be useful to identify bottlenecks when the demand on, or resources of, a component of the system is stressed. The model developed in this phase attempts to reduce the use of conservative estimates and instead increase fidelity in terms of representing infrastructure systems, ecosystems, and social institutions. Of course, introducing a more realistic model can also raise issues regarding how these components are 
represented; some observational data should be available at this stage for a simple validation of the model.

$$
\text { 2.2.2. Methods }
$$

Tier II tools may include matrices of resilience performance that utilize metrics from Tier I approaches but disaggregate them into sub-domains and time stages such as the Functional Resonance Analysis Method (FRAM) (Hollnagel, 2012) or the Resilience Matrix (Fox-Lent, Bates, and Linkov 2015). Decision analysis methods, such as multi-criteria decision analysis and other structured decision approaches, provide an appropriate set of tools to evaluate scenarios, allowing an understanding of how change leads to gains or losses in the system and the impact (or lack thereof) from a particular investment (Linkov and Moberg 2011). The sequence of assessment from Tier I to Tier II provides transparency and documentation so that any conclusions from the studies can be understood in light of what the model did and did not include and the reasons for those modeling choices.

\subsubsection{Outcomes}

The primary outcome of Tier II is a model that reveals the structure of the system and its interrelated components to support comparison of projects or investments to improve resilience. Some scenario analysis of potential future events can be performed, where stakeholders and experts define how management or policy scenarios are selected and how specific environmental and community parameters are integrated and weighted to describe system responses (such as by Thorisson et al. 2016). Such a model can be used to compare resilience management alternatives that are not mutually exclusive to obtain the best outcome across the system. This may be sufficient to initiate the resilience improvement process or make investment decisions for currently available funding. If the parallel Tier II risk assessment determines that significant residual risk remains but that the degree of uncertainty undermines the utility of a Tier II assessment, the system-wide resilience strategies will 
enhance overall performance beyond what would be obtained simply through risk mitigation measures. To determine whether additional work is warranted, a data assessment may also be carried out in order to verify data availability and fitness for purpose (quality). This can be done in concert with any data assessment necessary for a Tier III risk assessment. As with risk analysis, a tradeoff evaluation between the cost of new data collection and the benefit of the resilience assessment is performed to set the analysis boundaries.

\subsection{Tier VI: Networked System}

\subsubsection{Approach}

As is often the case in risk assessment, the user might frequently stop after Tier II and only move on to the time and effort of Tier III when the situation is appropriately complex or variable or uncertainty high enough that the learning in Tier II is insufficient to inform any actions. Tier III models seek to provide the highest fidelity in modeling a real-world system and thus observe the specific conditions under which risks arise or critical function performance drops. At this tier, the analysis can be very similar, if not coincident with, a Tier III risk assessment. The approach should consider interactions in ecological and technological components of the system along with an analysis of the impact of management decisions on affected social institutions and vulnerable populations (Moser and Ekstrom 2010). Here a full range of scenarios can be tested to better understand system performance in an uncertain future, as the model only requires the mode of failure, not the cause. Tier III can be prospective in predicting the performance of different system configurations under chronic and episodic disturbance.

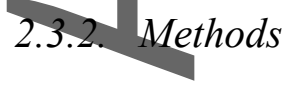

Possible approaches include system dynamics, graph theory, Bayesian networks, and agentbased models (Ganin et al., 2016; Ganin et al., 2017; Schultz and Smith, 2016; Boston et al., 
2014; Gao et al., 2016) that allow scenario analysis as well as Monte Carlo simulation to support sensitivity analysis. While many system managers may prefer to have the information provided by a high-quality Tier III model before making decisions, the time, cost, and data requirements frequently make this infeasible, and following the sequence of performing a Tier I and Tier II assessment may provide enough relevant insight to act.

\subsubsection{Outcomes}

The goal of a Tier III assessment is to reveal — to the extent possible — the risk to sustained critical function performance to the level that effective risk management plans can be developed. Ideally this process also includes modeling of the post-disruption recovery process in order to identify intervention opportunities that reduce downtime and the potential for spillover impacts to other sections. Either sensitivity analysis or scenario analysis can provide a range of potential performance results so that resilience interventions that are robust to a range of possible futures can be developed. In this way, the modeling and use requirements for Tier III resilience assessment merge with those for Tier III risk assessment.

\section{Discussion and Policy Implications}

In risk assessment, the approach is primarily sequential: first risk assessment is performed, then risk management strategies are developed. In resilience assessment, we believe that management alternatives should be considered iteratively through each stage in the analysis to reduce unnecessary analysis that is not directly associated with management actions. Globally or nationally accepted thresholds to characterize high or low resilience do not yet exist, but the regulatory community should not let that constrain early support for resilience assessment efforts. While this may seem like a departure from common regulatory implementations of risk assessment, a prime consideration for any new approach to assess and manage risks to infrastructural and natural systems is how such an approach interfaces 
with existing regulatory and policy requirements and capabilities. Presidential Policy

Directive 21 (PPD-21 2013) and Executive Order 13636 (EO-13636 2013) required agencies in the US federal government to explore resilience within various risk management activities pertaining to critical infrastructure. Rather than proposing tiered resilience as a stand-alone, fully novelconcept, we argue instead that such a method works as a complement to existing risk management tools and capacities in many agencies. This should help streamline the resilience adoption process, drive the call for new tools to fill gaps in complexity and system learning, and will reduce the overall burden of these executive guidelines.

We recommend that the tiered resilience approach integrate work from the many agencies, organizations, and researchers who have built resilience indices, visualization tools, and modeling methods. For a relatively simple system, significantly improved resilience may be achievable with a smaller toolbox where indices are sufficient to describe the system and potential vulnerabilities, and conservative estimates can address the uncertainties (see Quinlan et al., 2015 and Rosati et al., 2015 for a review of methods). Considerations of funding and time remain lower in Tier I as compared with Tier III, where a more complex system may require probabilistic and modeling across a range of future scenarios as well as cross-disciplinary analysis (e.g. Kott and Linkov, 2018; Ganin et al., 2016; Ayyub, 2014; and Boston et al., 2014). To supplement, rather than duplicate risk assessment, resilience may be best assessed with non-probabilistic methods, for example, possibility theory (Dubois and Prade, 1998; Schafer, 1987). The emphasis is on the fact that the main event will happen given an indefinite time horizon; the actual probability of this event is not the driving concern for resilience management. We recommend that adaptive management approaches (Allen and Garmestani, 2015) inform all tiers, allowing for management of the system while systematically reducing uncertainties regarding system response to both management change 
and system disruptions. This perspective may also prove valuable to agencies and organizations at all levels. Lacking standards, user input should be explicitly integrated to maximize the utility of an assessment. Managers and experts are needed in more advanced analysis to accurately describe the system organization and performance. Stakeholders can identify goals, priorities, and acceptable tradeoffs in performance (Cauffman, 2015) and thus guide resilience management toward effective and efficient solutions.

The benefits of the tiered approach are that each tier has a set of actionable items, but users can also move incrementally between the tiers as more detailed analysis is needed. Users can assess their system at each level, incorporate available data and stakeholder input on acceptability of performance, and then determine if the model employed is sufficiently accurate to describe the system and scenario. The tiered approach enables users to extract a range of responses from basic but practical, to sophisticated and predictive, in an effort to quantify the tactical steps needed to enhance resilience. Groups that seek an integrated strategy for assessing and communicating resilience, one that incorporates science into decision-making while working with limited funding, data, and timelines, may find this tiered approach yields a practical means of addressing pressing issues in a changing climate.

We acknowledge that many questions still remain. Uncertainty is a key driver in selecting risk analysis tools and the tiered structure for risk analysis helps to provide formal guidance on balance the cost of assessment against the potential reduction in uncertainty to find a practical management strategy. In the approach presented here, we view resilience management through a framework for making decisions on how to maintain critical functions and services during and after a disruptive event but it will be a feature of the methods adopted at each tier to manage uncertainty. The lack of quantitative guidance on when is it appropriate 
to move to the next tier of analysis is also a limitation; however, we believe that by adopting a tiered framework for resilience assessment, regulatory agencies can signal the need for specific methods to address existing gaps in tool availability and to have the output of the tools align such that thresholds for acceptable and unacceptable resilience can be developed. Though such an approach may not be immediately applicable to all regulatory agencies in the United States and elsewhere, it does interface well with existing capacities and requirements for traditional risk management for several organizations in a manner that allows for an iterative and adaptive approach to system resilience analysis within an environment of uncertainty and potentially shifting priorities.

\section{Acknowledgments}

The authors would like to thank the Aspen Global Change Institute for hosting the Risk and Resilience in the Face of Global Change workshop in December 2015 from which these ideas came together, specifically Susanne Moser. We appreciate funding for the workshop by NOAA and The Kresge Foundation. We would also like to acknowledge attendees of the aforementioned workshop, who as a group helped facilitate productive discussion that helped clarify these ideas. The Nebraska Cooperative Fish and Wildlife Research Unit is jointly supported by a cooperative agreement between the U.S. Geological Survey, the Nebraska Game and Parks Commission, the University of Nebraska-Lincoln, the United States Fish and Wildlife Service and the Wildlife Management Institute. This work was supported in part by the US Army Corps of Engineers. The view expressed here are those by authors and do not represent views of the US Government agencies or sponsoring organizations.

\section{References}


Allen, C. R., \& Garmestani, A. S. (2015). Adaptive management. In Adaptive Management of Social-Ecological Systems (pp. 1-10). Springer, Dordrecht.

American Society for Testing and Materials (ASTM) (2015). Standard Guide for Risk-Based Corrective Aetion. E2081-00, ASTM International, West Conshohocken, PA.

Angeler, D. G., \& Allen, C. R. (2016). Quantifying resilience. Journal of Applied Ecology, $53(3), 617-624$.

Ayyub, B. M. (2014). Systems resilience for multihazard environments: Definition, metrics, and valuation for decision making. Risk Analysis, 34(2), 340-355.

Arnott, J. C., Moser, S. C., \& Goodrich, K. A. (2016). Evaluation that counts: A review of climate change adaptation indicators \& metrics using lessons from effective evaluation and science-practice interaction. Environmental Science \& Policy, 66, 383392.

Bakkensen, L. A., Fox- Lent, C., Read, L. K., \& Linkov, I. (2017). Validating resilience and vulnerability indices in the context of natural disasters. Risk analysis, 37(5), 9821004.

Boston, M., Liu, Z., Jacques, C., \& Mitrani-Reiser, J. (2014). Towards assessing the resilience of a community in seismic events using agent based modeling. Network for ( $)$ Earthquake Engineering Simulation.

Cauffman, S. A. (2015). Community Resilience Planning Guide for Buildings and Infrastructure Systems. NIST Special Publication 1190. Washington DC.

Cutter, S. L., Burton, C. G., \& Emrich, C. T. (2010). Disaster resilience indicators for benchmarking baseline conditions. Journal of Homeland Security and Emergency Management, 7(1).

Dubois, D., \& Prade, H. (2012). Possibility theory. In Computational complexity (pp. 22402252). Springer New York. 
Executive Order 13636 (EO-13636). (2013). Improving critical infrastructure cybersecurity. The White House, Washington DC.

Federal Emergency Management Agency (FEMA) 310. (1998). Handbook for the seismic evaluation of buildings - A prestandard. Washington, DC: American Society of Civil Engineers.

Flanagan, B. E., Gregory, E. W., Hallisey, E. J., Heitgerd, J. L., \& Lewis, B. (2011). A social vulnerability index for disaster management. Journal of homeland security and emergency management, $8(1)$.

Florin, M.-V. and Linkov, I. (Eds.) (2016), IRGC Resource Guide on Resilience. Retrieved from: https://www.irgc.org/risk-governance/resilience/.

Fox-Lent, C., Bates, M. E., \& Linkov, I. (2015). A matrix approach to community resilience assessment: an illustrative case at Rockaway Peninsula. Environment Systems and Decisions, 35(2), 209-218.

Gallopín, G. C. (2006). Linkages between vulnerability, resilience, and adaptive capacity. Global environmental change, 16(3), 293-303.

Ganin, A. A., Massaro, E., Gutfraind, A., Steen, N., Keisler, J. M., Kott, A., Mangoubi, R. \& Linkov, I. (2016). Operational resilience: concepts, design and analysis. Scientific reports, 6, 19540 .

Ganin, A. A., Kitsak, M., Marchese, D., Keisler, J. M., Seager, T., \& Linkov, I. (2017). Resilience and efficiency in transportation networks. Science advances, 3(12),

e1701079.

Gao, J., Barzel, B., \& Barabási, A. L. (2016). Universal resilience patterns in complex networks. Nature, 530(7590), 307.

Gott, D. (2012). Risk Assessment Paradigm. European Food Safety Authority. Stakeholder Workshop 21 September 2012, Brussels. 
Hollnagel, E. (2012). FRAM, the functional resonance analysis method: modelling complex socio-technical systems. Ashgate Publishing, Ltd..

Kott, A. \& Linkov, I., (Eds.). (2018). Cyber Resilience of Systems and Networks. Springer, Amsterdam.

Linkov, I. and Fox-Lent, C. (2016). "A Tiered Approach to Resilience Assessment” in IRGC Resourde guide on resilience (Eds. Linkov, I. and Florin, M.V.). Retrieved from: https://www.irgc.org/risk-governance/resilience/.

Larkin, S., Fox-Lent, C., Eisenberg, D. A., Trump, B. D., Wallace, S., Chadderton, C., \& Linkov, I. (2015). Benchmarking agency and organizational practices in resilience decision making. Environment Systems and Decisions, 35(2), 185-195.

Linkov, I., \& Moberg, E. (2011). Multi-criteria decision analysis: environmental applications and case studies. CRC Press.

Linkov, I., \& Palma-Oliveira, J. M. (Eds.). (2017). Resilience and risk: Methods and application in environment, cyber and social domains. Springer, Amsterdam.

Moser, S. C., \& Boykoff, M. T. (Eds.). (2013). Successful adaptation to climate change: Linking science and policy in a rapidly changing world. Routledge.

Moser, S. C., \& Ekstrom, J. A. (2010). A framework to diagnose barriers to climate change adaptation. Proceedings of the national academy of sciences, 107(51), 22026-22031.

National Research Council. (1983). Risk assessment in the federal government: managing the process. National Academies Press.

U.S. Indian Ocean Tsunami Warning System Program (USIOTWSP). (2007). How Resilient is your coastal community? A guide for evaluating coastal community resilience to tsunamis and other hazards. U.S. Indian Ocean Tsunami Warning System Program supported by the United States Agency for International Development and partners, Bangkok, Thailand. 
Nemec, K. T., J. Chan, C. Hoffman, T. L. Spanbauer, J. A. Hamm, C. R. Allen, T. Hefley, D. Pan, and P. Shrestha. 2013. Assessing resilience in stressed watersheds. Ecology and Society 19(1): 34 .

Nordgren, J., Stults, M., \& Meerow, S. (2016). Supporting local climate change adaptation:

Where we are and where we need to go. Environmental Science \& Policy, 66, 344-

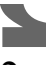

352.

Özkaynak, H., Frey, H. C., \& Hubbell, B. (2008). Characterizing variability and uncertainty

in exposure assessments improves links to environmental decision-making. EM (Pittsburgh, Pa.), 58(7), 18.

Peacock, W. G., Brody, S. D., Seitz, W. A., Merrell, W. J., Vedlitz, A., Zahran, S., Harriss,

R. C., \& Stickney, R. (2010). Advancing Resilience of Coastal Localities:

Developing, Implementing, and Sustaining the Use of Coastal Resilience Indicators:

A Final Report. Hazard Reduction and Recovery Center. Texas A\&M University.

College Station, $\mathrm{Tx}$.

Presidential Policy Directive 21 (PPD-21). (2013). Critical infrastructure security and

resilience. The While House. Washington DC.

Quinlan, A. E., Berbés- Blázquez, M., Haider, L. J., \& Peterson, G. D. (2016). Measuring and assessing resilience: broadening understanding through multiple disciplinary perspectives. Journal of Applied Ecology, 53(3), 677-687.

Rosati, J. D., Touzinsky, K. F., \& Lillycrop, W. J. (2015). Quantifying coastal system resilience for the US Army Corps of Engineers. Environment Systems and Decisions, 35(2), 196-208.

Sands, D. (2015). An innovative scorecard for evaluating resiliency in our cities. Planet@ Risk, 3(1). 
Shafer, G. (1987). Belief functions and possibility measures. The analysis of fuzzy information, 1, 51-84.

Schultz, M. T., \& Smith, E. R. (2016). Assessing the resilience of coastal systems: A probabilistic approach. Journal of Coastal Research, 32(5), 1032-1050.

Thorisson, H., Lambert, J. H., Cardenas, J. J., \& Linkov, I. (2017). Resilience analytics with application to power grid of a developing region. Risk Analysis, 37(7), 1268-1286.

Williams, P. R., Nolan, M., \& Panda, A. (2014). Disaster Resilience Scorecard for Cities. UNISDR. http://www.unisdr.org/2014/campaigncities/Resilience $\% 20$ Scorecard\%20V1.5.pdf

United Nations (2015). Sendai framework for disaster risk reduction 2015-2030.

A/CONF.224/CRP.1 Third United Nations World Conference on Disaster Risk Reduction, 18 March 2015.

U.S. Environmental Protection Agency (USEPA) (2014). Risk Assessment Forum White Paper:Probabilistic Risk Assessment Methods and Case Studies. EPA/100/R09/001A. Washington, D.C.: Risk Assessment Forum, Office of the Science Advisor,

\section{USEPA}

U.S. Nuclear Regulatory Commission (USNRC) (2009). An approach for plant-specific, riskinformed decision making: technical specifications. Regulatory Guide 1.177.

Washington DC.

Walker, B., Holling, C. S., Carpenter, S., \& Kinzig, A. (2004). Resilience, adaptability and transformability in social-ecological systems. Ecology and society, 9(2).

Woods, D. D. (2006). Essential characteristics of resilience. Resilience engineering: Concepts and precepts, 21-34. 
World Health Organization (WHO) (2008). Part 1: guidance document on characterizing and communicating uncertainty in exposure assessment. World Health Organization, Geneva Switzerland.

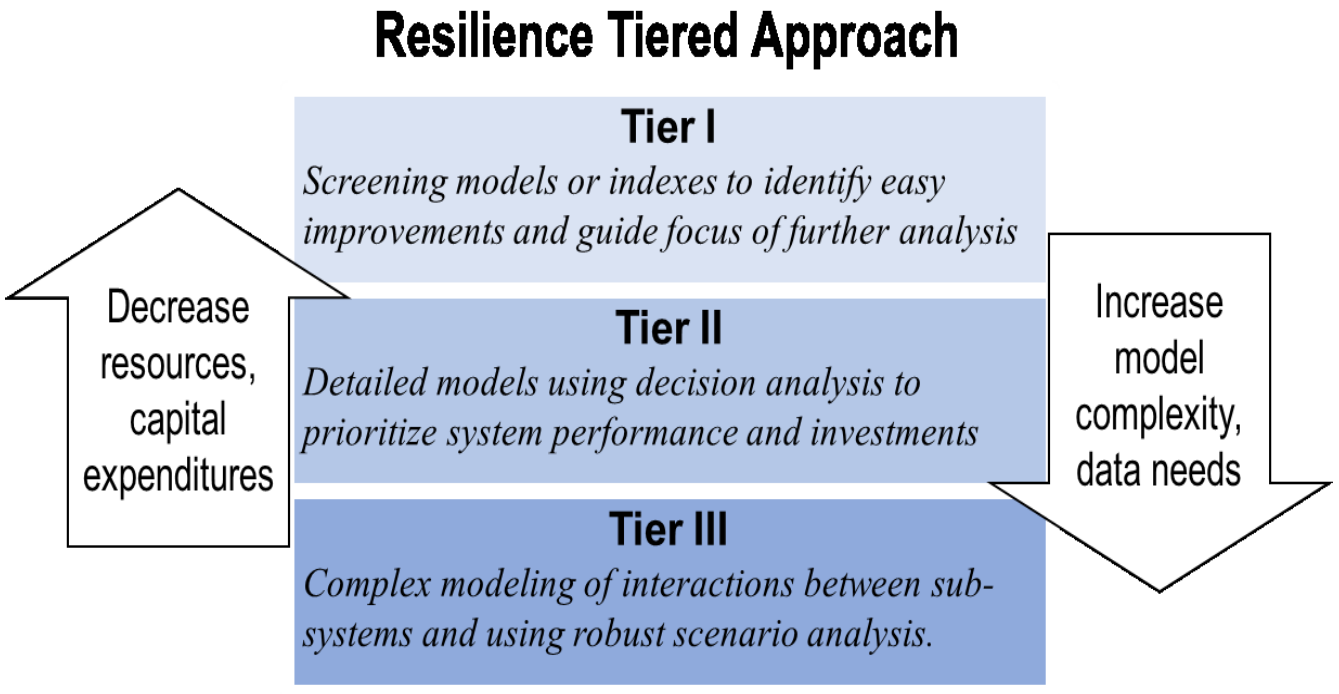

Figure 1. Overview of tiered approach to resilience assessment

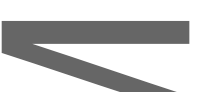

Risk Assessment

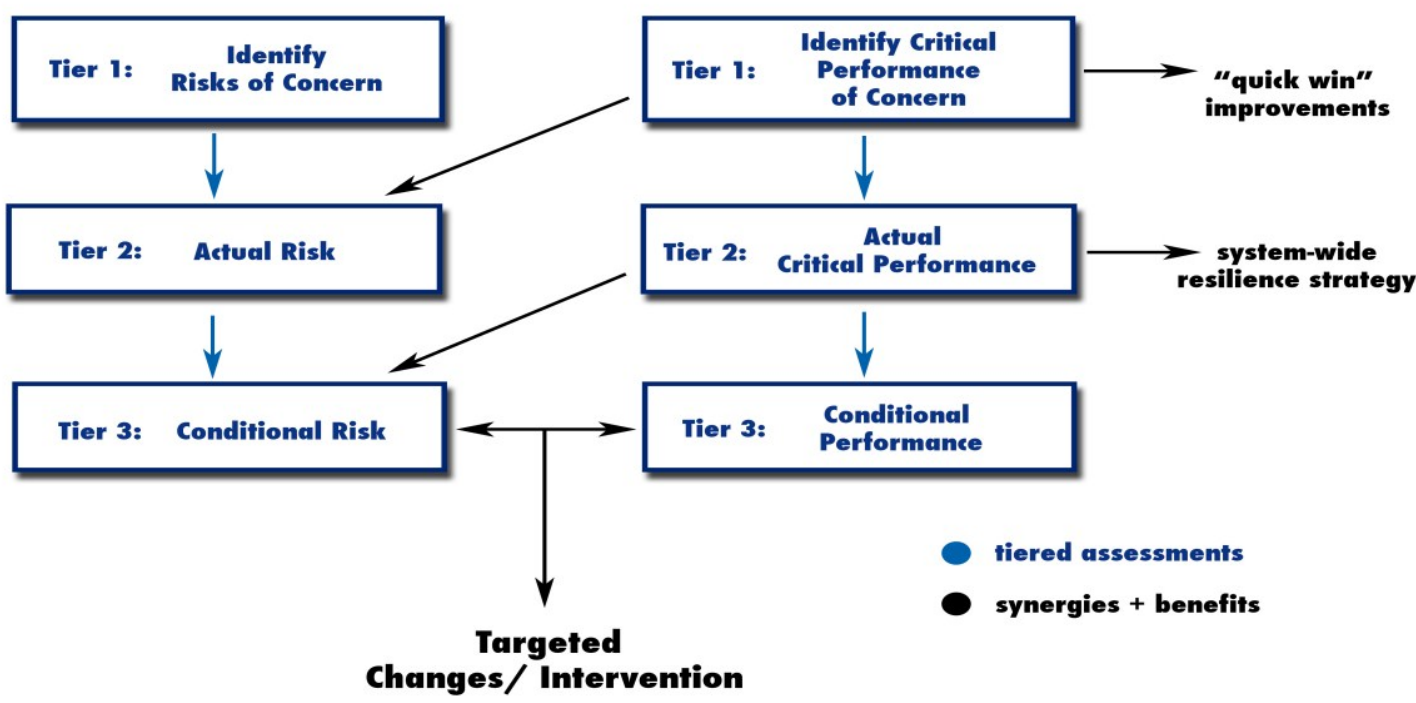

Figure 2. Integrated workflow of Risk and Resilience assessments. 